\title{
ĐÁNH GIÁ Độ CHÍNH XÁC CỦA MÔ HÌNH QUASIGEOID EGM2008 TRÊN LÃNH THỔ VIẸT NAM
}

\author{
PGS. TSKH. HÀ MINH HÒA \\ Viện Khoa học Đo đạc và Bản đồ
}

\section{Tóm tắt:}

Bài báo khoa học này đã đánh giá độ chính xác của mô hình quasigeoid trọng lực cục bộ được xây dựng từ mô hình EGM2008 và được chuyển về mặt quasigeoid Hòn Dấu. Khi so sánh với mô hình quasigeoid GPS/thủy chuẩn cục bộ được xây dụng dụa trên 185 giá trị dị thường độ cao GPS/thủy chuẩn của 185 điểm độ cao hạng I quốc gia, độ chính xác của mô hình quasigeoid trọng lực cục bộ ở mức $\pm 8,5 \mathrm{~cm}$ độ chênh nhỏ nhất giữa dị thường độ cao GPS/thủy chuẩn và dị thường độ cao trọng lực bằng - 0,224m, còn độ chênh lớn nhất bằng $0,236 m$.

\section{1. Đặt vấn đề}

Mô hình trọng trường Quả đất EGM2008 được Tổ chức Tri thức - Địa không gian (NGA - National Geospatial Agency) của Mỹ phát triển vào năm 2008 (Pavlis Nikolas K, Simon A. Holmes, Steve C. Kenyon, John K. Factor (2008)). Do các giá trị dị thường độ cao (hoặc độ cao geoid) được xác định từ mô hình EGM2008 được coi là tương thích với các giá trị dị thường độ cao GNSS/thủy chuẩn (hoặc độ cao geoid GNSS/thủy chuẩn), nên nhiều quốc gia trên thế giới đã nghiên cứu và công bố các kết quả đánh giá sự tương thích nêu trên. Điều này rất quan trọng trong việc định hướng sử dụng mô hình EGM2008 để xây dựng mô hình quasigeoid (hoặc geoid) quốc gia. Để tiện sử dụng tiếp theo, chúng ta gọi mặt quasigeoid (hoặc mặt geoid) được xác định từ EGM2008 là mặt quasigeoid EGM2008 (hoặc mặt geoid EGM2008), còn mặt quasigeoid (hoặc mặt geoid) được xác định từ các giá trị dị thường độ cao GNSS/thủy chuẩn (hoặc độ cao geoid GNSS/thủy chuẩn) là mặt quasigeoid GNSS/thủy chuẩn (hoặc mặt geoid GNSS/thủy chuẩn), thêm vào đó dị thường độ cao (hoặc độ cao Geoid) được xác định từ mặt quasigeoid EGM2008 (hoặc mặt geoid EGM2008) được gọi là dị thường độ cao trọng lực (hoặc độ cao Geoid trọng lực).

Do các hệ độ cao dựa trên mặt biển trung bình tại một trạm nghiệm triều (được gọi là trạm nghiệm triều 0 ) của các nước được xây dựng từ rất lâu, thêm vào đó các độ cao chuẩn (hoặc độ cao chính) của các mốc độ cao quốc gia không được thường xuyên hiệu chỉnh bởi sự chuyển dịch đứng của vỏ Trái đất, nên việc đánh giá sự tương tương thích của mặt quasigeoid EGM2008 (hoặc mặt geoid EGM2008) với mặt quasigeoid GNSS/thủy chuẩn (hoặc mặt geoid GNSS/thủy chuẩn) gặp rất nhiều khó khăn. Ở Việt Nam cũng gặp những khó khăn tương tự do các tuyến độ cao hạng $\mathrm{I}$, II quốc gia bắt đầu được xây dựng từ thập niên 70 của thế kỷ $X X$ và không được hiệu chỉnh do sự chuyển dịch đứng của vỏ Trái đất.

Ở nước Mỹ, nhờ các kết quả đo lặp thủy chuẩn đã phát hiện sự dịch chuyển đứng (nâng lên, lún) của các mốc độ cao quốc gia dưới tác động của hoạt động kiến tạo của vỏ Trái đất. Theo tài liệu (Hastie L.M. and Savage J.C. (1970)), động đất ở Alaska vào năm 1964

Người phản biện: TS. Nguyễn Đình Thành 
đã gây ra chuyển động kiến tạo ở Anchorage và làm bề mặt Quả đất nâng lên đến $0,55 \mathrm{~m}$. Theo tài liệu (Pelton J.R. and Smith R.B. (1979)), tốc độ nâng lên của công viên quốc gia Yellowstone đạt đến $14 \mathrm{~mm} / 1$ năm. Tại vùng đồng bằng Diablo ở bang New Mexico, trong giai đoạn từ 1953 - 1977 tốc độ nâng đạt mức 4 mm/1 năm (Reilinger R.E., Oliver J.E., Brown L.D., Sanford A.R. and Balazs E.I. (1980)). Tại lưu vực sông Saugus gần khu vực đứt gãy San Gabriel đã quan sát được độ lún ở mức gần $9 \mathrm{~m}$ trong giai đoạn 1953 - 1964 (Reilinger R.E. (1980)).

Tại khu vực giữa Kakegawa và Omalzaki ở vùng Tokai (Nhật Bản), nhờ các kết quả đo GPS liên tục đã phát hiện sự thay đổi của độ cao trắc địa với tốc độ - $6 \mathrm{~mm} / 1$ năm trong thời gian 3,5 năm từ tháng $1 / 1995$ đến tháng $7 / 1999$. Từ các kết quả đo lặp thủy chuẩn ở khu vực trên đã xác định được tốc độ thay đổi của độ cao chính ở mức - 4,5 mm/ 1 năm (Minoru Tanaka (2000)).

Tại Vương quốc Anh, theo tài liệu (Iliffe J.C., Ziebart M., Cross P.A., Forsberg R., Strykowski G., Tscherning C.C. (2003)), việc so sánh các hiệu độ cao Geoid GPS/thủy chuẩn và độ cao Geoid trọng lực trên 179 điểm độ cao cơ sở cho thấy tồn tại các sai số hệ thống ở mức $0,510 \mathrm{~m}$ theo hướng Bắc - Nam. Do các mạng lưới độ cao quốc gia của nước Anh được xây dựng trong nhiều giai đoạn 1912 - 1921, 1936 - 1952 và 1952 - 2956, sự trồi, lún của các mốc độ cao cơ sở được gây ra bởi hoạt động kiến tạo của vỏ Trái đất và hoạt động khai thác mỏ.

Do đó, các nước đều xác định hạn sai của các hiệu giữa dị thường độ cao GNSS/thủy chuẩn và dị thường độ cao trọng lực (hoặc các hệ giữa độ cao geoid GNSS/thủy chuẩn và độ cao geoid trọng lực) và chỉ lựa chọn các điểm độ cao nhà nước có các hiệu nêu trên thỏa mãn hạn sai để đánh giá sự tương thích của mặt quasigeoid EGM2008 (hoặc mặt geoid EGM2008) với mặt quasigeoid GNSS/thủy chuẩn (hoặc mặt geoid GNSS/thủy chuẩn).

Các thử nghiệm trên thế giới cho thấy ở nhiều quốc gia, mô hình Quageoid (hoặc Geoid khi sử dụng độ cao chính) được xác định từ mô hình trọng trường Quả đất EGM2008 và mạng lưới độ cao hạng I, II quốc gia có sự tương thích nhau. Ví dụ, theo tài liệu (Thomas Gruber (2008)), kết quả so sánh dị thường độ cao GPS/thủy chuẩn trên 675 điểm ở CHLB Đức với dị thường độ cao được xác định từ mô hình EGM2008 cho thấy sai số trung phương của hiệu các dị thường độ cao nêu trên ở mức $\pm 3,8 \mathrm{~cm}$, độ chênh cực tiểu băng $-8,8 \mathrm{~cm}$, còn độ chênh cực đại bă̆ng $13,3 \mathrm{~cm}$. Theo kết quả nghiên cứu tại Australia (Claessens S.J., Featherstone W.E., Anjasmara I.M., Filmer M.S. (2009)), sai số trung phương độ chênh giữa dị thường độ cao trọng lực được xác định từ mô hình AUSGEOID98 và dị thường độ cao trọng lực được xác định từ mô hình EGM2008 bằng $0,164 \mathrm{~m}$. Độ chênh hệ thống giữa dị thường độ cao GPS/ thủy chuẩn và dị thường độ cao trọng lực được xác định từ mô hình EGM2008 trên 1013 điểm GPS/thủy chuẩn dọc theo Australia là $-0,025 \mathrm{~m}$, trên 2435 điểm GPS/thủy chuẩn ở Tây Australia là $-0,060 \mathrm{~m}$, trên 45 điểm GPS/thủy chuẩn ở Nam Australia là $-0,036 \mathrm{~m}$.

Tuy nhiên ở nhiều quốc gia khác, mô hình Quageoid (hoặc Geoid khi sử dụng độ cao chính) được xác định từ mô hình trọng trường Quả đất EGM2008 và mạng lưới độ cao hạng I, II quốc gia không tương thích nhau. Theo tài liệu (Gomaa M. Dawod, Hoda F. Mohamed, and Sherine S. Ismail. (2010)), các kêt quả xác định dị thường độ cao theo phương pháp GPS/thủy chuẩn trên 207 điểm và độ cao Geoid từ mô hình EGM2008 tại 
thung lũng Bắc sông Nil và đồng bằng ở Ai Cập cho thấy sai số trung phương của các độ chênh của các dị thường độ cao bằng $\pm 0,187 \mathrm{~m}$. Độ chênh của dị thường độ cao GPS/thủy chuẩn so với độ cao Geoid được xác định từ mô hình EGM2008 đều ở mức -1,76 m.

Đối với Thổ Nhĩ Kỳ, theo các kết quả nghiên cứu trong (Ibrahim Yilmaz, Mustafa Yilmaz, Mevlut Gullu, Bayram Turgut. (2010)), dựa trên 313 điểm GPS/thủy chuẩn thuộc mạng lưới GPS cơ sở quốc gia Thổ Nhĩ Kỳ (khoảng 600 điểm) thực hiện so sánh dị thường độ cao GPS/thủy chuẩn với dị thường độ cao trọng lực được xác định từ mô hình EGM2008 cho thấy hiệu trung bình của dị thường độ cao GPS/thủy chuẩn và dị thường độ cao trọng lực được xác định từ mô hình EGM2008 ở mức \pm 0,294 m. Theo tài liệu (Metin Soycan (2014)), khi so sánh dị thường độ cao được xác định từ mô hình EGM2008 với các giá trị dị thường độ cao GPS/thủy chuẩn, độ chênh nhỏ nhất bằng $-0,888 \mathrm{~m}$ và độ chênh cực đại bằng $0,242 \mathrm{~m}$, còn khi so sánh dị thường độ cao được xác định từ mô hình EGM2008 với mô hình quasigeoid của Thổ Nhĩ Kỳ TG03, độ chênh nhỏ nhất bằng $0,271 \mathrm{~m}$ và độ chênh cực đại bằng $0,753 \mathrm{~m}$.

Ở Trung Quốc đã xây dựng mạng lưới khống chế dị thường độ cao HACN2000 được xác định nhờ công nghệ GPS và được chia thành 2 hạng: hạng $A$ trùng với mạng lưới GPS hạng $\mathrm{A}$ và trùng với các điểm độ cao hạng I, II quốc gia bao gồm 30 điểm với khoảng cách trung bình $700 \mathrm{~km}$, còn hạng $B$ trùng với mạng lưới GPS hạng $B$ và các điểm độ cao hạng II, III bao gồm 750 điểm với mật độ điểm trung bình ở phần Đông Trung Quốc là $80 \mathrm{~km}$, ở phần Trung tâm là $130 \mathrm{~km}$ và ở phần Tây Trung Quốc là $250 \mathrm{~km}$ (China National Report on Geodesy (1999-2002)). Trong tài liệu (Li Jiancheng, Chao Dingbo, Shen Wenbin, Zang Shoujian, Zou Xiancai, Jiang Weiping and Yao Yibin (2011)), khi sử dụng 652 điểm GPS/thủy chuẩn từ mạng lưới HACN2000 các hạng $\mathrm{A}$, B để so sánh với mô hình EGM2008 cho thấy sai số trung phương của các độ chênh ở mức $\pm 0,284 \mathrm{~m}$, còn khi sử dụng 1812 điểm GPS/thủy chuẩn từ các điểm thuộc mạng lưới HACN2000 các hạng $\mathrm{A}, \mathrm{B}$ và các điểm độ cao thuộc 7 lưới độ cao của các tỉnh cho thấy sai số trung phương của các độ chênh ở mức $\pm 0,222 \mathrm{~m}$, còn các độ chênh nằm trong khoảng từ $\pm 0,375 \mathrm{~m}$ đến $\pm 0,680$ $\mathrm{m}$.

Việt Nam còn gặp một khó khăn khác liên quan đến việc sử dụng mô hình EGM2008: Các dữ liệu đo trọng lực chi tiết ở Việt Nam không được sử dụng để xây dựng mô hình EGM2008. Khi thành lập mô hình EGM2008, ở các khu vực không có các dữ liệu trọng lực chi tiết được ký hiệu bởi thuật ngữ "Fill - in", trong đó có Việt Nam. Trong trường hợp này, tổ chức NGA đã sử dụng mô hình DTM2006.0 2' x 2' để tạo mặt độ cao trung bình (mặt quy chiếu) và sử dụng mô hình global DTM 2006.0 giải 30" x 30" để tạo ra mô hình global 2' x 2' RTM $\Delta g$. Từ mô hình này đã khai triển điều hòa đến mức 2700 (Pavlis N.K., Factor J.K. and Holmes S.A. (2007)). Do Việt Nam nằm trong khu vực "Fill-in" của mô hình EGM2008, nên các dữ liệu dị thường trọng lực trong các ô chuẩn $5^{\prime}$ x $5^{\prime}$ được xác định theo phương pháp RTM (Residual Terrain Model). Theo tài liệu (Pavlis N.K., Holmes S.A., Kenyon S.C. and Factor J.K. (2009)), đánh giá chất lượng của các giá trị dị thường trọng lực trong các ô chuẩn 5 ' x 5' trong khu vực "Fill-in" như sau:

- Sai số ủy nhiệm (commission error) của dị thường độ cao (hoặc độ cao Geoid) (được gây ra do hệ số khai triển điều hòa chưa được làm chính xác vì không có các dữ liệu trọng lực chi tiết) nằm ở mức $\pm(15-22) \mathrm{cm}$;

- Dị thường trọng lực còn dư trong các ô chuẩn 5’ x 5’ ở mức từ -8 mGal đến -16 mGal, 
còn sai số chung của các giá trị dị thường trọng lực trong các ô chuẩn $5^{\prime}$ x $5^{\prime}$ ở $^{\prime}$ mức \pm (910) $\mathrm{mGal}$.

Theo tài liệu (Jekeli C., Yanh H.J. and Kwon J.H. (2009)), do không tính đến tần số cao của trọng trường, nên dị thường độ cao được xác định từ mô hình EGM2008 còn chứa sai số bỏ qua (omission error).

Đối với lãnh thổ Việt Nam, do không hiệu chỉnh độ cao chuẩn của các độ cao hạng I, II quốc gia do sự chuyển dịch đứng của vỏ Trái đất và không sử dụng các dữ liệu đo trọng lực chi tiết để xây dựng mô hình EGM2008, các độ chênh của mặt quasigeoid EGM2008 và mặt quasigeoid GNSS/thủy chuẩn có độ lớn là bao nhiêu?. Bài toán khoa học này sẽ trả lời câu hỏi này.

\section{Giải quyết vấn đề}

Mặt khởi tính cho hệ độ cao HP72 ở Việt Nam là mặt quasigeoid quốc gia trùng với mặt geoid cục bộ Hòn Dấu tại trạm nghiệm triều Hòn Dấu và càng tách dần khỏi mặt geoid cục bộ Hòn Dấu khi càng đi sâu vào đất liền. Chúng ta gọi mặt quasigeoid này là mặt quasigeoid cục bộ. Khoảng cách giữa mặt quasigeoid cục bộ so với mặt ellipsoid WGS84 quốc tế theo đường vuông góc với mặt ellipsoid này tương ứng với điểm cần quan tâm được gọi là dị thường độ cao GPS/thủy chuẩn của điểm. Khi đo GPS trên các điểm độ cao hạng I quốc gia và xử lý các dữ liệu GPS trong ITRF tương ứng với ellipsoid WGS84 quốc tế, dị thường độ cao GPS/thủy chuẩn $\xi_{\Sigma}$ trong hệ triều 0 (zero - tide system) của điểm độ cao hạng I bất kỳ được xác định theo công thức:

$$
\zeta_{z}=\bar{H}_{z}-H_{z}^{\gamma},
$$

ở đây $\bar{H}_{z}$ - độ cao trắc địa của điểm tương ứng với ellipsoid WGS84 quốc tế và trong hệ triều $0 ; H_{z}^{\gamma}$ - độ cao chuẩn của điểm trong hệ triều 0 (xem hình 1 ở dưới đây).

Việc chuyển độ cao trắc địa trong hệ không phụ thuộc triều được xác định từ kết quả xử lý dữ liệu đo GPS trong ITRF về hệ triều 0 và chuyển độ cao chuẩn từ hệ triều trung bình về hệ triều 0 được nghiên cứu chi tiết trong tài liệu (Hà Minh Hòa và nnk (2012a)). Do mặt quasigeoid cục bộ hoàn toàn được xác định nhờ phương pháp đo GPS/thủy chuẩn, nên mặt quasigeoid này còn được gọi là mặt quasigeoid GPS/thủy chuẩn cục bộ.

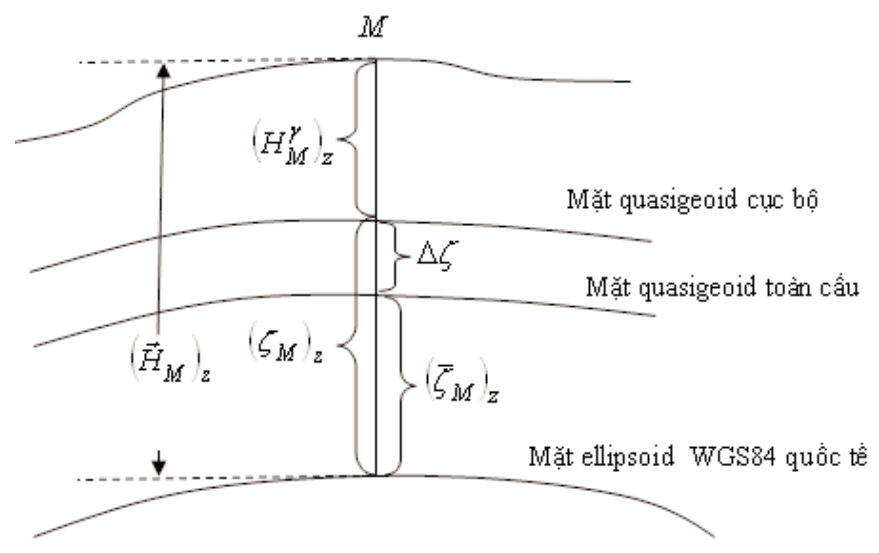

Hinh 1 
Chúng ta gọi mặt quasigeoid toàn cầu là mặt quasigeoid trùng với mặt geoid toàn cầu trên các biển và các đại dương, càng tách dần khỏi mặt geoid toàn cầu khi càng đi sâu vào đất liền. Dị thường độ cao trọng lực $\bar{\zeta}_{z}$ trong hệ triều 0 của điểm độ cao hạng I bất kỳ được xác định từ mô hình EGM2008 tương ứng với mặt quasigeoid toàn cầu và được chuyển về mặt quasigeoid cục bộ theo công thức:

$$
\hat{\bar{\zeta}}=\bar{\zeta}_{z}+\Delta \zeta
$$

ở đây $\Delta \zeta$ - độ cao của mặt quasigeoid cục bộ so với mặt quasigeoid toàn cầu tương ứng với vị trí của điểm độ cao hạng I (xem hình 1 ở trên).

Theo các kết quả nghiên cứu đã được công bố trong các tài liệu (Hà Minh Hòa và nnk (2012a); Hà Minh Hòa (2012b); Hà Minh Hòa (2013a)), đại lượng $\Delta \zeta=0.890 \mathrm{~m}$ và không đổi trên toàn bộ lãnh thổ Việt Nam.

Dị thường độ cao trọng lực $\hat{\bar{\zeta}}_{z}$ (2) tương ứng với mặt quasigeoid trọng lực cục bộ. Như vậy, việc đánh giá sự tương thích của mặt quasigeoid EGM2008 với mặt quasigeoid GNSS/thủy chuẩn thực chất là việc đánh giá sự tương thích của mặt quasigeoid GPS/thủy chuẩn cục bộ với mặt quasigeoid trọng lực cục bộ. Đối với điểm độ cao hạng I bất kỳ, hiệu giữa dị thường độ cao $\zeta_{x}$ (1) và dị thường độ cao $\hat{\bar{\zeta}}_{z}$ (2) được ký hiệu bằng $d=\zeta_{z}-\dot{\bar{\zeta}}_{z}$. Hạn sai của độ lệch $d$ được đánh giá theo công thức:

$$
\left(m_{d}\right)_{\text {gioinan }}=2,0 \cdot \sqrt{m_{\zeta}^{2}+m_{\hat{\zeta}}^{2}},
$$

ở đây $m_{\zeta}$ - sai số trung phương của dị thường độ cao GPS/thủy chuẩn; $m_{\overline{\bar{\zeta}}}$ - sai số trung phương của dị thường độ cao trọng lực.

Sai số trung phương của dị thường độ cao GPS/thủy chuẩn được đánh giá theo công thức:

$$
m_{\zeta}=\sqrt{m_{\bar{H}}^{2}+m_{H}^{2}} \text {. }
$$

Khi nhận sai số trung phương của độ cao trắc địa $m_{\bar{I}} \approx 2 \mathrm{~cm}$, sai số trung phương lớn nhất của độ cao chuẩn hạng $\mathrm{I} m_{H^{\gamma}}=6,0 \mathrm{~cm}$, sai số trung phương của dị thường độ cao GNSS/thủy chuẩn được đánh giá bằng $m_{\zeta}=6,32 \mathrm{~cm}$. Theo tài liệu (Pavlis N.K., Holmes S.A., Kenyon S.C. and Factor J.K. (2009)), trên phạm vi toàn cầu sai số trung phương của dị thường độ cao trọng lực $m_{\hat{\hat{\zeta}}}=10,3 \mathrm{~cm}$, hạn sai của hiệu $d_{i}=\left(\zeta_{i}\right)_{z}-\left(\hat{\bar{\zeta}}_{i}\right)_{z}$ được đánh giá theo công thức (3) có giá trị:

$$
\left(m_{d}\right)_{\text {gioihan }}=2,0 \cdot \sqrt{m_{\zeta}^{2}+m_{\hat{\zeta}}^{2}}=0,240 \mathrm{~m} \text {. }
$$

Việc kiểm tra sự có mặt của các sai số hệ thống trong các hiệu $d_{i}$ được thực hiện nhờ điều kiện: 


$$
\left|\sum_{i=1}^{N} d_{i}\right| \leq 0,25 \cdot \sum_{i=1}^{N}\left|d_{i}\right| .
$$

Nếu điều kiện trên thỏa mãn, thì trong dãy các hiệu $d_{i}$ không có các sai số hệ thống.

Đối với Việt Nam, nhóm nghiên cứu của đề tài này đã thu nhập được các dữ liệu xử lý kết quả đo GPS trong ITRF2005 và các độ cao chuẩn của 230 điểm thủy chuẩn hạng I nhà nước được chọn làm các điểm GPS/thủy chuẩn từ kết quả thực hiện Thiết kế kỹ thuật - dự toán "Xây dựng mô hình Geoid địa phương trên lãnh thổ Việt Nam (Hạng mục GPS-TC và Trọng lực)" thuộc Dự án Chính phủ "Thành lập cơ sở dữ liệu nền thông tin địa lý ở tỷ lệ 1/10.000 gắn với mô hình số độ cao" của Cục Đo đạc và Bản đồ Việt Nam trong những năm 2009 - 2010. Các kết quả phân tích cho thấy:

- Có tất cả 74 điểm có các độ chênh $d_{i}$ với giá trị tuyệt đối vượt hạn sai (4) bao gồm các điểm I(BH-TH)18, I(BH-TH)19, I(BH-TH)25, I(BH-TH)30, I(BH-TH)36-1, I(BH-TH)39, I(BHTH)44, I(BH-TH)48, I(BH-TH)93-1, I(BH-TH)99, I(BH-TH)104-1, I(BH-TH)106, I(BHTH)140, I(BH-TH)144A, I(BH-TH)152, I(BH-TH)146, I(BT-APD)25, I(BT-APD)35, I(LSHN)22, I(LS-HN)29, I(LS-HN)36, I(BH-LS)9, I(BH-LS)11-1, I(BH-LS)16A, I(BH-LS)21, I(BHLS)31, I(BH-LS)36, I(BH-LS)41, I(BH-LS)44-1, I(BH-LS)48, I(DN-BMT)4, I(DN-BMT)8, I(DN-BMT)40, I(DN-BMT)47A, I(DN-BMT)52, I(HN-VL)4-1, I(HN-VL)6-1, I(HN-VL)10A, I(HN-VL)14-1, I(HN-VL)16A, I(HN-VL)28-1, I(HN-VL)19, I(VL-HT)14, I(VL-HT)209-1, I(VLHT)218, I(VL-HT)231, I(VL-HT)234-1, I(VL-HT)293, I(HN-HP)2A, I(HN-HP)5, I(HN-HP)7, I(HN-HP)11A, I(HN-HP)16, I(HP-NB)8-1, I(HP-NB)14A, I(BH-HN)33, I(BH-HN)39, I(BHHN)42, I(BH-HN)48, I(HP-MC)27, I(HN-VL)24-1, I(VL-HT)2, I(LS-TY)14, I(BH-HN)8, I(HPMC)39, I(VL-HT)227, I(BH-TH)131-1, I(BH-TH)128, I(BH-TH)149-1, I(VL-HT)222, I(VLHT)216, I(BH-TH)84, I(BH-HN)26, I(BH-TH)56, I(HP-NB)5.

Một số điểm độ cao hạng I có các độ chênh $d_{i}$ với giá trị tuyệt đối lớn hơn $0,4 \mathrm{~m}$ được trình bày ở bảng 1 ở dưới đây

\section{Bảng 1}

\begin{tabular}{|c|c|c|c|}
\hline Tên điểm & Độ chênh $(\mathrm{m})$ & Tên điểm & Độ chênh $(\mathrm{m})$ \\
\hline $\mathrm{I}(\mathrm{BH}-\mathrm{HN}) 33$ & 0,566 & $\mathrm{I}(\mathrm{HN}-\mathrm{VL}) 4-1$ & 0,580 \\
\hline $\mathrm{I}(\mathrm{BH}-\mathrm{HN}) 39$ & 0,571 & $\mathrm{I}(\mathrm{HN}-\mathrm{VL}) 6-1$ & 0,581 \\
\hline $\mathrm{I}(\mathrm{BH}-\mathrm{HN}) 42$ & 0,543 & $\mathrm{I}(\mathrm{HN}-\mathrm{VL}) 10 \mathrm{~A}$ & 0,464 \\
\hline $\mathrm{I}(\mathrm{BH}-\mathrm{HN}) 48$ & 0,680 & $\mathrm{I}(\mathrm{LS}-\mathrm{HN}) 22$ & 0,440 \\
\hline $\mathrm{I}(\mathrm{HN}-\mathrm{HP}) 2 \mathrm{~A}$ & 0,670 & $\mathrm{I}(\mathrm{LS}-\mathrm{HN}) 29$ & 0,512 \\
\hline $\mathrm{I}(\mathrm{HN}-\mathrm{HP}) 5$ & 0,704 & $\mathrm{I}(\mathrm{LS}-\mathrm{HN}) 36$ & 0,599 \\
\hline $\mathrm{I}(\mathrm{HN}-\mathrm{HP}) 7$ & 0,616 & $\mathrm{I}(\mathrm{HP}-\mathrm{NB}) 14 \mathrm{~A}$ & 0,435 \\
\hline $\mathrm{I}(\mathrm{HN}-\mathrm{HP}) 11 \mathrm{~A}$ & 0,401 & $\mathrm{I}(\mathrm{BH}-\mathrm{TH}) 99$ & 0,820 \\
\hline $\mathrm{I}(\mathrm{DN}-\mathrm{BMT}) 47 \mathrm{~A}$ & $-0,492$ & & \\
\hline
\end{tabular}


Như vậy, trong tổng số 230 kết quả đo GPS/thủy chuẩn được Cục Đo đạc và Bản đồ Việt Nam đo trên 230 điểm độ cao hạng I trong giai đoạn 2009 - 2010, chúng ta chỉ sử dụng được 156 điểm hạng I (chiếm $67.83 \%$ ) có hiệu $d_{i}=\left(\zeta_{i}\right)_{z}-\left(\hat{\bar{\zeta}}_{i}\right)_{z}$ thỏa mãn hạn sai (4) để đánh giá mô hình Quasigeoid ở Việt Nam (xem bảng 2 ở dưới đây).

Trong khuôn khổ thực hiện công trình (Hà Minh Hòa và nnk (2012a)), Viện Khoa học Đo đạc và Bản đồ đã đo kiểm tra GPS 4 ngày đêm liên tục trên 9 điểm độ cao hạng I từ ngày 31/03/2012 đến hết ngày 02/05/2012. Các điểm độ cao hạng I này bị che khuất, không đo được GPS, nên từ các điểm này dẫn độ cao ra các điểm phụ đảm bảo các điều kiện thu được tín hiệu vệ tinh GPS. Các điểm phụ bao gồm LS1 (đo nối từ điểm I(LS-TY)7 đến I(LSTY)8A), TB01 (đo nối từ điểm I(HP-NB)14A đến I(HP-NB)13), TH01 (đo nối từ điểm I(BH$\mathrm{TH}) 144 \mathrm{~A}$ đến I(BH-TH)143), QN01 (đo nối từ điểm I(VL-HT) 68 đến I(VL-HT)70A), QNG1 (đo nối từ điểm I(VL-HT)89 đến I(VL-HT)90A), PY01 (đo nối từ điểm I(VL-HT)128A) đến I(VL-HT)129), KH01 (đo nối từ điểm I(VL-HT)154A đến I(VL-HT)152-5), BT01 (đo nối từ điểm I(VL-HT)226 đến I(VL-HT)225A), BP01 (đo nối từ điểm I(BMT-APD)52 đến I(BMT$\mathrm{AHD}) 51 \mathrm{~A})$. Các kết quả tính toán cho thấy các hiệu $d_{i}=\left(\zeta_{i}\right)_{z}-\left(\hat{\bar{\zeta}}_{i}\right)_{z}$ trên 04 điểm LS01, TH01, KH01 và BT01 vượt hạn sai (4). Do đó, chúng ta chỉ sử dụng được 5 điểm để đánh giá mô hình Quasigeoid của biển Đông và vùng đồng bằng, trung $\mathrm{du}$ (xem Bảng 2 ở dưới đây).

Trong năm 2013, Viện Khoa học Đo đạc và Bản đồ đã tiến hành đo kiểm tra thêm 30 điểm độ cao hạng I quốc gia nằm dọc bờ biển Việt Nam trong khoảng thời gian từ tháng 7 đến tháng 08/2013. Xuất phát từ giả định rằng các mốc độ cao hạng I quốc gia nằm ở khu vực bị che khuất sẽ ít bị xê dịch do các hoạt động kinh tế - xã hội (cải tạo, mở rộng các đường quốc lộ, xây dựng các công trình .v.v...), các mốc độ cao hạng I được chọn ở các khu vực bị che khuất, không cho phép thu các tín hiệu từ các vệ tinh GNSS. Từ các mốc độ cao hạng I quốc gia này dẫn độ cao bằng các máy thủy chuẩn điện tử đến các vị trí thoáng đãng (các mốc phụ) nằm gần các mốc để xác định độ cao của các mốc phụ và tiến hành đo GNSS tại các mốc phụ được xây dựng (xem trong Bảng 2 ở dưới đây). Các dữ liệu đo GPS trên các mốc phụ trong 04 ngày đêm được xử lý trong ITRF2008 tương ứng với ellipsoid WGS84 quốc tế bằng phần mềm Bernese v. 5.2. Các kết quả tính toán cho thấy trong tổng số 30 mốc phụ, chỉ có 24 mốc phụ (chiếm 80\%) đảm bảo các hiệu $d_{i}=\left(\zeta_{i}\right)_{z}-\left(\hat{\bar{\zeta}}_{i}\right)_{z}$ thỏa mãn hạn sai (4). Các mốc độ cao hạng I có khả năng bị xê dịch do 
hoạt động kiến tạo của vỏ Trái đất bao gồm I(HP-NB)02, I(HP-NB)10, I(HP-NB)15, I(HN$\mathrm{VL}) 17, \mathrm{I}(\mathrm{HN}-\mathrm{VL}) 29-1, \mathrm{I}(\mathrm{VL}-\mathrm{HT}) 30$. Các kết quả tính các hiệu $d_{i}=\left(\zeta_{i}\right)_{z}-\left(\hat{\bar{\zeta}}_{i}\right)_{z}$ đạt hạn sai của 24 mốc phụ được đưa vào bảng 2 để tham gia đánh giá độ chính xác của mô hình Quasigeoid trên lãnh thổ Việt Nam.

Như vậy, trong tổng số 269 điểm hạng I được sử dụng để đánh giá độ chênh $d_{i}=\left(\zeta_{i}\right)_{z}-\left(\hat{\bar{\zeta}}_{i}\right)_{z}$ chúng ta chỉ nhận được 185 điểm (chiếm 68,77\%) có các độ chênh thỏa mãn hạn sai (4) và được đưa vào bảng 2 ở dưới đây. Việc kiểm tra sự có mặt của các sai số hệ thống trong chuỗi 185 hiệu $d_{i}=\left(\zeta_{i}\right)_{z}-\left(\hat{\zeta}_{i}\right)_{z}$ trong bảng 2 theo tiêu chuẩn (5) cho bất đẳng thức ở dưới đây:

$$
\left|\sum_{i=1}^{185} d_{i}\right|=2,874 \leq 0,25 \cdot \sum_{i=1}^{185}\left|d_{i}\right|=0,25 x 18,015=4,504 .
$$

Sự thỏa mãn tiêu chuẩn (5) xác định sự không tồn tại của các sai số hệ thống trong chuỗi 185 hiệu $d_{i}=\left(\zeta_{i}\right)_{z}-\left(\hat{\bar{\zeta}}_{i}\right)_{z}$ trong bảng 2 .

Khi coi dị thường độ cao trọng lực và dị thường độ cao GNSS/thủy chuẩn có cùng độ chính xác, sai số trung phương dị thường độ cao được đánh giá bằng:

$$
m_{\zeta}= \pm \sqrt{\frac{\left[d_{i}^{2}\right]}{2 \cdot N}}= \pm \sqrt{\frac{2,7134205721}{2 \times 185}}= \pm 0,085 \mathrm{~m}
$$

Như vậy, khi dựa vào 185 điểm độ cao hạng I có các độ chênh $d_{i}=\left(\zeta_{i}\right)_{z}-\left(\hat{\bar{\zeta}}_{i}\right)_{z}$ thỏa mãn hạn sai (4), chúng ta thấy rằng độ chính xác của mặt quasigeoid trọng lực cục bộ được xác định từ mô hình EGM2008 theo công thức (2) so với mặt quasigeoid GPS/thủy chuẩn cục bộ được xác định từ các giá trị dị thường độ cao GPS/thủy chuẩn trên 185 điểm nêu trên nằm ở mức $\pm 8,5 \mathrm{~cm}$.

\begin{tabular}{|c|c|c|c|c|c|}
\hline $\begin{array}{c}\text { STT } \\
\text { i }\end{array}$ & Tên điểm & $\begin{array}{c}\text { Hiệu } \\
d_{i}=\left(\zeta_{i}\right)_{z}-\left(\hat{\bar{\zeta}}_{i}\right)_{z} \\
\text { (m) }\end{array}$ & $\begin{array}{c}\text { STT } \\
\text { i }\end{array}$ & Tên điểm & $\begin{array}{c}\text { Hiệu } \\
d_{i}=\left(\zeta_{i}\right)_{z}-\left(\hat{\bar{\zeta}}_{i}\right)_{z} \\
\text { (m) }\end{array}$ \\
\hline \multicolumn{6}{|c|}{ Các kết quả đo của Cục Đo đạc và Bản đồ Việt Nam giai đoạn 2009 - 2010} \\
\hline 1 & $\mathrm{I}(\mathrm{BH}-\mathrm{LS}) 97$ & 0,093 & 17 & I(LS-HN)22 & $-0,117$ \\
\hline 2 & $\mathrm{I}(\mathrm{BH}-\mathrm{TH}) 122 \mathrm{~A}$ & 0,006 & 18 & $\mathrm{I}(\mathrm{LS}-\mathrm{HN}) 29$ & $-0,045$ \\
\hline
\end{tabular}

\section{Bảng 2}


Nghiên cứu

\begin{tabular}{|c|c|c|c|c|c|}
\hline 3 & I(BMT-APD)30 & 0,159 & 19 & I(LS-HN)36 & 0,042 \\
\hline 4 & $\mathrm{I}(\mathrm{BH}-\mathrm{TH}) 119$ & 0,026 & 20 & $\mathrm{I}(\mathrm{VL}-\mathrm{HT}) 325-1$ & 0,075 \\
\hline 5 & $\mathrm{I}(\mathrm{BH}-\mathrm{HN}) 33$ & 0,009 & 21 & $\mathrm{I}(\mathrm{BH}-\mathrm{HN}) 16 \mathrm{~A}$ & 0,073 \\
\hline 6 & $\mathrm{I}(\mathrm{BH}-\mathrm{HN}) 39$ & 0,014 & 22 & I(HN-VL)28-1 & 0,008 \\
\hline 7 & $\mathrm{I}(\mathrm{BH}-\mathrm{HN}) 42$ & $-0,014$ & 23 & $\mathrm{I}(\mathrm{HN}-\mathrm{VL}) 95$ & $-0,162$ \\
\hline 8 & $\mathrm{I}(\mathrm{BH}-\mathrm{HN}) 48$ & 0,123 & 24 & $\mathrm{I}(\mathrm{HN}-\mathrm{VL}) 45-1$ & 0,040 \\
\hline 9 & I(HN-HP)11A & $-0,156$ & 25 & $\mathrm{I}(\mathrm{VL}-\mathrm{HT}) 152-1$ & $-0,046$ \\
\hline 10 & I(HN-HP)2A & 0,113 & 26 & I(VL-HT)121 & 0,059 \\
\hline 11 & $\mathrm{I}(\mathrm{HN}-\mathrm{HP}) 5$ & 0,147 & 27 & I(VL-HT) 73 & 0,172 \\
\hline 12 & $\mathrm{I}(\mathrm{HN}-\mathrm{VL}) 10 \mathrm{~A}$ & $-0,093$ & 28 & I(VL-HT)95 & 0,154 \\
\hline 13 & $\mathrm{I}(\mathrm{HN}-\mathrm{HP}) 7$ & 0,059 & 29 & I(HN-VL)23-1 & $-0,171$ \\
\hline 14 & I(HN-VL)4-1 & 0,023 & 30 & I(DN-BT)35 & $-0,181$ \\
\hline 15 & I(HN-VL)6-1 & $-0,006$ & 31 & I(HN-VL)97 & $-0,175$ \\
\hline 16 & I(HP-NB)14A & $-0,122$ & 32 & I(VL-HT)269 & $-0,176$ \\
\hline 33 & I(VL-HT)192 & $-0,174$ & 67 & $\mathrm{I}(\mathrm{BH}-\mathrm{TH}) 5$ & $-0,038$ \\
\hline 34 & I(HN-VL)32-1 & $-0,158$ & 68 & I(HN-VL)38-1 & $-0,042$ \\
\hline 35 & $\mathrm{I}(\mathrm{VL}-\mathrm{HT}) 284 \mathrm{~A}$ & $-0,172$ & 69 & I(VL-HT)197 & $-0,055$ \\
\hline 36 & $\mathrm{I}(\mathrm{BH}-\mathrm{TH}) 1-2$ & $-0,142$ & 70 & I(BT-APD)63 & $-0,055$ \\
\hline 37 & I(VL-HT)43 & $-0,155$ & 71 & I(VL-HT)127-3 & $-0,049$ \\
\hline 38 & I(HN-VL)86 & $-0,147$ & 72 & I(BT-APD)59-1 & $-0,052$ \\
\hline 39 & I(VL-HT)200-1 & $-0,157$ & 73 & I(VL-HT)278-1 & $-0,046$ \\
\hline 40 & I(VL-HT)313 & $-0,157$ & 74 & I(VL-HT)108 & $-0,038$ \\
\hline 41 & I(HP-C) 9 & $-0,135$ & 75 & I(DN-BT)77 & $-0,035$ \\
\hline 42 & I(VL-HT)64 & $-0,141$ & 76 & I(BT-NH)17-1 & $-0,038$ \\
\hline 43 & $\mathrm{I}(\mathrm{VL}-\mathrm{HT}) 273 \mathrm{~A}$ & $-0,142$ & 77 & $\mathrm{I}(\mathrm{BH}-\mathrm{TH}) 122 \mathrm{~A}$ & $-0,020$ \\
\hline 44 & I(DN-BT)67 & $-0,133$ & 78 & I(VL-HT) 83 & $-0,032$ \\
\hline 45 & I(DN-BT)18-1 & $-0,108$ & 79 & $\mathrm{I}(\mathrm{BH}-\mathrm{HN}) 17$ & $-0,017$ \\
\hline 46 & I(BT-APD)46 & $-0,111$ & 80 & $\mathrm{I}(\mathrm{HN}-\mathrm{VL}) 45-1$ & $-0,022$ \\
\hline 47 & I(DN-BT)16 & $-0,097$ & 81 & I(BH-TH)65 & $-0,008$ \\
\hline 48 & I(VL-HT)305 & $-0,107$ & 82 & I(VL-HT)178 & $-0,022$ \\
\hline 49 & $\mathrm{I}(\mathrm{DN}-\mathrm{BT}) 28$ & $-0,091$ & 83 & I(VL-HT)103 & $-0,015$ \\
\hline 50 & I(VL-HT) 150 & $-0,095$ & 84 & I(HN-VL)64 & $-0,006$ \\
\hline
\end{tabular}


Nghiên cúu

\begin{tabular}{|c|c|c|c|c|c|}
\hline 51 & $\mathrm{I}(\mathrm{VL}-\mathrm{HT}) 152-1$ & $-0,088$ & 85 & $\mathrm{I}(\mathrm{VL}-\mathrm{HT}) 141-3$ & $-0,014$ \\
\hline 52 & $\mathrm{I}(\mathrm{HN}-\mathrm{VL}) 34-1$ & $-0,072$ & 86 & $\mathrm{I}(\mathrm{VL}-\mathrm{HT}) 329 \mathrm{~A}$ & $0-, 014$ \\
\hline 53 & $\mathrm{I}(\mathrm{HP}-\mathrm{MC}) 48 \mathrm{~A}$ & $-0,068$ & 87 & $\mathrm{I}(\mathrm{HN}-\mathrm{VL}) 72$ & 0,001 \\
\hline 54 & $\mathrm{I}(\mathrm{BH}-\mathrm{TH}) 3-1$ & $-0,044$ & 88 & $\mathrm{I}(\mathrm{VL}-\mathrm{HT}) 158$ & 0,000 \\
\hline 55 & $\mathrm{I}(\mathrm{VL}-\mathrm{HT}) 181$ & $-0,084$ & 89 & $\mathrm{I}(\mathrm{VL}-\mathrm{HT}) 121$ & 0,007 \\
\hline 56 & $\mathrm{I}(\mathrm{LS}-\mathrm{TY}) 4$ & $-0,060$ & 90 & $\mathrm{I}(\mathrm{DN}-\mathrm{BT}) 74$ & 0,022 \\
\hline 57 & $\mathrm{I}(\mathrm{VL}-\mathrm{HT}) 309 \mathrm{~A}$ & $-0,081$ & 91 & $\mathrm{I}(\mathrm{BH}-\mathrm{LS}) 88-1$ & 0,024 \\
\hline 58 & $\mathrm{I}(\mathrm{VL}-\mathrm{HT}) 317$ & $-0,076$ & 92 & $\mathrm{I}(\mathrm{VL}-\mathrm{HT}) 98$ & 0,009 \\
\hline 59 & $\mathrm{I}(\mathrm{VL}-\mathrm{HT}) 187$ & $-0,072$ & 93 & $\mathrm{I}(\mathrm{BH}-\mathrm{LS}) 85-1$ & 0,028 \\
\hline 60 & $\mathrm{I}(\mathrm{VL}-\mathrm{HT}) 170-1$ & $-0,071$ & 94 & $\mathrm{I}(\mathrm{BH}-\mathrm{LS}) 93$ & 0,026 \\
\hline 61 & $\mathrm{I}(\mathrm{HP}-\mathrm{MC}) 41$ & $-0,042$ & 95 & $\mathrm{I}(\mathrm{BH}-\mathrm{LS}) 71$ & 0,031 \\
\hline 62 & $\mathrm{I}(\mathrm{VL}-\mathrm{HT}) 130$ & $-0,058$ & 96 & $\mathrm{I}(\mathrm{BT}-\mathrm{APD}) 56$ & 0,011 \\
\hline 63 & $\mathrm{I}(\mathrm{HN}-\mathrm{VL}) 56$ & 0,028 & 97 & $\mathrm{I}(\mathrm{VL}-\mathrm{HT}) 73$ & 0,117 \\
\hline 64 & $\mathrm{I}(\mathrm{BH}-\mathrm{TH}) 11$ & 0,041 & 98 & $\mathrm{I}(\mathrm{VL}-\mathrm{HT}) 159-3$ & 0,116 \\
\hline 65 & $\mathrm{I}(\mathrm{HN}-\mathrm{VL}) 40-1$ & 0,034 & 99 & $\mathrm{I}(\mathrm{VL}-\mathrm{HT}) 262 \mathrm{~A}$ & 0,127 \\
\hline 66 & $\mathrm{I}(\mathrm{BH}-\mathrm{LS}) 77$ & 0,043 & 100 & $\mathrm{I}(\mathrm{BH}-\mathrm{HS}) 68$ & 0,154 \\
\hline
\end{tabular}

\begin{tabular}{|c|c|c|c|c|c|}
\hline 101 & $\mathrm{I}(\mathrm{VL}-\mathrm{HT}) 87$ & 0,028 & 129 & $\mathrm{I}(\mathrm{BT}-\mathrm{APD}) 6$ & 0,137 \\
\hline 102 & $\mathrm{I}(\mathrm{VL}-\mathrm{HT}) 247 \mathrm{~A}$ & 0,022 & 130 & $\mathrm{I}(\mathrm{VL}-\mathrm{HT}) 250$ & 0,140 \\
\hline 103 & $\mathrm{I}(\mathrm{LS}-\mathrm{TY}) 1$ & 0,042 & 131 & $\mathrm{I}(\mathrm{BT}-\mathrm{NH}) 9$ & 0,149 \\
\hline 104 & $\mathrm{I}(\mathrm{VL}-\mathrm{HT}) 325-1$ & 0,023 & 132 & $\mathrm{I}(\mathrm{BH}-\mathrm{LS}) 82$ & 0,178 \\
\hline 105 & $\mathrm{I}(\mathrm{DN}-\mathrm{BT}) 83$ & 0,029 & 133 & $\mathrm{I}(\mathrm{HN}-\mathrm{VL}) 68$ & 0,174 \\
\hline 106 & $\mathrm{I}(\mathrm{VL}-\mathrm{HT}) 78$ & 0,032 & 134 & $\mathrm{I}(\mathrm{DN}-\mathrm{BT}) 89$ & 0,171 \\
\hline 107 & $\mathrm{I}(\mathrm{LS}-\mathrm{HN}) 7$ & 0,055 & 135 & $\mathrm{I}(\mathrm{VL}-\mathrm{HT}) 254 \mathrm{~A}$ & 0,172 \\
\hline 108 & $\mathrm{I}(\mathrm{VL}-\mathrm{HT}) 71$ & 0,051 & 136 & $\mathrm{I}(\mathrm{BH}-\mathrm{TH}) 88$ & 0,194 \\
\hline 109 & $\mathrm{I}(\mathrm{BH}-\mathrm{TH}) 59$ & 0,074 & 137 & $\mathrm{I}(\mathrm{BT}-\mathrm{APD}) 3$ & 0,183 \\
\hline 110 & $\mathrm{I}(\mathrm{VL}-\mathrm{HT}) 173-2$ & $-0,056$ & 138 & $\mathrm{I}(\mathrm{DN}-\mathrm{BT}) 96$ & 0,189 \\
\hline 111 & $\mathrm{I}(\mathrm{BH}-\mathrm{TH}) 70 \mathrm{~A}$ & 0,075 & 139 & $\mathrm{I}(\mathrm{BH}-\mathrm{LS}) 6-1$ & $-0,232$ \\
\hline 112 & $\mathrm{I}(\mathrm{HN}-\mathrm{VL}) 50$ & 0,070 & 140 & $\mathrm{I}(\mathrm{BH}-\mathrm{HN}) 4-1$ & $-0,222$ \\
\hline 113 & $\mathrm{I}(\mathrm{VL}-\mathrm{HT}) 123$ & 0,064 & 141 & $\mathrm{I}(\mathrm{HP}-\mathrm{MC}) 37$ & $-0,223$ \\
\hline 114 & $\mathrm{I}(\mathrm{LS}-\mathrm{HN}) 12$ & 0,079 & 142 & $\mathrm{I}(\mathrm{HP}-\mathrm{MC}) 24$ & $-0,221$ \\
\hline
\end{tabular}


Nghiên cứu

\begin{tabular}{|c|c|c|c|c|c|}
\hline 115 & I(HP-C)4-1 & 0,085 & 143 & I(VL-HT)238A & $-0,231$ \\
\hline 116 & I(BH-LS)80 & 0,087 & 144 & I(BT-APD)42 & $-0,235$ \\
\hline 117 & I(DN-BT)86 & 0,069 & 145 & I(VL-HT)61 & $-0,221$ \\
\hline 118 & I(VL-HT)320A & 0,067 & 146 & I(HP-MC) 12 & $-0,211$ \\
\hline 119 & I(BH-LS)97 & 0,093 & 147 & I(HP-MC) 16 & $-0,208$ \\
\hline 120 & I(BH-LS)94-1 & 0,104 & 148 & I(VL-HT)95 & $-0,213$ \\
\hline 121 & I(BT-APD)49-1 & 0,092 & 149 & I(VL-HT)48 & $-0,210$ \\
\hline 122 & I(HN-VL)76 & 0,111 & 150 & I(BT-NH)22 & $-0,206$ \\
\hline 123 & I(VL-HT)95 & 0,106 & 151 & I(DN-BT)59 & $-0,200$ \\
\hline 124 & I(VL-HT)113 & 0,105 & 152 & I(BH-HN)9-1 & $-0,234$ \\
\hline 125 & I(LS-HN)10 & 0,122 & 153 & I(DN-BT)100 & 0,205 \\
\hline 126 & I(BH-HN)19-1 & 0,124 & 154 & I(LS-HN)19 & 0,224 \\
\hline 127 & I(BT-NH)11-1 & 0,109 & 155 & I(BH-TH)80 & 0,235 \\
\hline 128 & I(BH-HN)20-1 & 0,126 & 156 & I(BT-APD)1-2 & 0,236 \\
\hline
\end{tabular}

\begin{tabular}{|c|c|c|c|c|c|}
\hline \multicolumn{5}{|c|}{ Viện Khoa học Đo đạc và Bản đồ đo năm 2012 } \\
\hline 157 & TB01 & $-0,120$ & & & \\
\hline 158 & QN01 & 0,029 & & & \\
\hline 159 & QNG1 & 0,109 & & & \\
\hline 160 & PY01 & 0,109 & & & \\
\hline 161 & BP01 & $-0,002$ & & & \\
\hline \multicolumn{5}{|c|}{ Viện Khoa học Đo đạc và Bản đồ đo năm 2013 } \\
\hline 162 & I(HP-MC)45 & 0,122 & 174 & HT73 & 0,128 \\
\hline 163 & MC26 & $-0,224$ & 175 & HT84 & 0,042 \\
\hline 164 & MC10 & $-0,210$ & 176 & HT94 & 0,090 \\
\hline 165 & NB18 & $-0,237$ & 177 & HT106 & 0,005 \\
\hline 166 & $22 A 1$ & $-0,033$ & 178 & HT121 & $-0,057$ \\
\hline 167 & $38 A 1$ & $-0,102$ & 179 & HT127-4 & $-0,009$ \\
\hline 168 & VL48 & 0,008 & 180 & HT141-3 & 0,040 \\
\hline 169 & VL59 & $-0,023$ & 181 & HT159-1 & $-0,059$ \\
\hline 170 & VL73 & 0,115 & 182 & HT173-3 & $-0,055$ \\
\hline 171 & VL93 & $-0,214$ & 183 & HT188 & $-0,146$ \\
\hline 172 & HT03 & $-0,218$ & 184 & HT197 & 0,056 \\
\hline 173 & HT58 & $-0,190$ & 185 & HT216 & $-0,172$ \\
\hline
\end{tabular}




\section{Kết luận và kiến nghị}

Về nguyên tắc, chúng ta có thể sử dụng các giá trị $\left(\zeta_{i}\right)_{z},\left(\hat{\bar{\zeta}}_{i}\right)_{z}$ trên 185 điểm trong bảng 2 để làm tương thích (fitting) mặt quasigeoid trọng lực cục bộ được xác định từ mô hình EGM2008 theo công thức (2) với mặt quasigeoid GPS/thủy chuẩn cục bộ theo phương pháp collocation phương sai để xây dựng mô hình quasigeoid quốc gia. Tuy nhiên tồn tại nguyên nhân chính cản trở việc xây dựng mô hình quasigeoid độ chính xác cao ở Việt Nam: Do mô hình quasigeoid trọng lực cục bộ được xác định từ mô hình EGM2008 theo công thức (2), nên mặt quasigeoid trọng lực cục bộ song song và có cùng cấu trúc với mặt quasigeoid toàn cầu từ EGM2008. Do chưa sử dụng các dữ liệu đo trọng lực chi tiết ở Việt Nam để hiệu chỉnh các hệ số khai triển điều hòa của mô hình EGM2008, nên mặt quasigeoid toàn cầu cũng như mặt quasigeoid trọng lực cục bộ ở Việt Nam phản ánh chưa chính xác các sóng chu kỳ ngắn của thế trọng trường Quả đất trên lãnh thổ Việt Nam. Điều này sẽ gây ra các sai số bị bỏ qua (omission errors) trong các giá trị dị thường độ cao được xác định;

Ngoài ra, độ chính xác của dị thường độ cao ở mức $\pm 8,5 \mathrm{~cm}$ là quá lớn khi đặt vấn đề xây dựng hệ quy chiếu không gian quốc gia. Yêu cầu độ chính xác được đặt ra phải cao hơn \pm 4 cm (Hà Minh Hòa (2013b)).

Do đó, việc phát triển công tác đo đạc trọng lực chi tiết và nghiên cứu phương pháp hiệu chỉnh các hệ số khai triển điều hòa của mô hình EGM2008 dựa trên các dữ liệu đo trọng lực chi tiết ở Việt Nam là những công việc cấp bách trong giai đoạn hiện nay.

Ngoài ra, như đã trình bày trong tài liệu (Hà Minh Hòa (2014)), việc bình sai mạng lưới độ cao hạng I, II quốc gia dựa trên mặt geoid cục bộ Hòn Dấu sẽ cho phép nhận được các tham số ẩn bình sai là các giá trị thế trọng trường của các mốc độ cao hạng I, II quốc gia. Từ đây, chúng ta xác định các giá trị thế nhiễu của các mốc độ cao hạng I, II quốc gia và sử dụng các giá trị này để hiệu chỉnh các hệ số khai triển điều hòa của mô hình EGM2008. O

\section{Tài liệu tham khảo}

[1]. China National Report on Geodesy (1999-2002) for the 23rd General Assembly of IUGG, Sapporo, Japan, June 30 - July 112003.

[2]. Claessens S.J., Featherstone W.E., Anjasmara I.M., Filmer M.S. (2009). In Australian data really validating EGM2008, or is EGM2008 just in/validating Australian data ?. Western Australian Centre for Geodesy \& the Institute for Geoscience Research. Curtin Univesity of Technology. (S.Claessens@curtin.edu.au; Featherstone@curtin.edu.au).

[3]. Gomaa M. Dawod, Hoda F. Mohamed, and Sherine S. Ismail. (2010). Evaluation and Adaptation of the EGM2008 Geopotential Model along the Northern Nile Valley, Egypt: Case Study. J. Surv. Engrg. Volume 136, Issue 1, pp. 36-40 (February 2010). 
[4]. Hastie L.M. and Savage J.C. (1970). A dislocation model for the Alaska earthquake. Bull. Seismol. Soc. Amer., V.60, p.1389-1392.

[5]. Hà Minh Hòa và nnk (2012a). Nghiên cứu cơ sở khoa học của việc hoàn thiện hệ độ cao gắn liền với việc xây dựng hệ tọa độ động lực quốc gia. Đề tài khoa học và công nghệ cấp Bộ Tài nguyền và Mổi trường giai đoạn 2010 - 2012. Hà Nội - 2012.

[6]. Hà Minh Hòa (2012b). Nghiên cứu xác định thế năng trọng trường thực $W_{0}$ của mặt Geoid cục bộ trùng với mặt biển trung bình tại trạm nghiệm triều Hòn Dấu. Báo cáo khoa học. Tuyển tập báo cáo Hội nghị Khoa học và Công nghệ "Trắc địa và Bản đồ vì sự nghiệp Tài nguyên và Môi trường". Viện Khoa học Đo đạc và Bản đồ - Hội Trắc địa, Bản đồ và Viễn thám Việt Nam. Hà Nội - Tháng 10/2012. Trg. 6-19.

[7]. Ha Minh Hoa (2013a). Estimating the geopotential value $\mathrm{W}_{0}$ of the local geoid based on data from local and global normal heights of GPS/Leveling points in Vietnam. Geodesy and Cartography. Taylor \& Francis. UDK 528.21, doi:10.3846/20296991.2013.823705, V.39 (3): 99-105.

[8]. Hà Minh Hòa (2013b). Các vấn đề liên quan đến việc xây dựng hệ quy chiếu không gian quốc gia. Tạp chí Khoa học Đo đạc và Bản đồ, No18, 12/2013, trg. 1 -9.

[9]. Hà Minh Hòa (2014). Hiệu chỉnh các hệ số điều hòa cầu của mô hình trọng trường Quả đất nhờ các kết quả bình sai mạng lưới độ cao hạng I, II quốc gia trong hệ độ cao dựa trên mặt Geoid cục bộ. Tạp chí Khoa học Đo đạc và Bản đồ, No19, 03/2014, trg. 1 -8.

[10]. Ibrahim Yilmaz, Mustafa Yilmaz, Mevlut Gullu, Bayram Turgut. (2010). Evaluation of recent global geopotential models based on GPS/levelling data over Afyonkarahisar (Turkey). Scientific Research and Essays. Vol. 5(5), pp. 484-493, 4 March 2010.

[11]. Iliffe J.C., Ziebart M., Cross P.A., Forsberg R., Strykowski G., Tscherning C.C. (2003). OSGM02: A New model for converting GPS-derived heights to local height datums in Great Britain and Ireland. Star - Science and Technology of Archelogical Research, Vol. 37, Issue 290, pp. 276 - 293.

[12]. Jekeli C., Yanh H.J. and Kwon J.H. (2009). Evaluation of EGM2008 - globally and locally in South Korea. In: Newton's Bulletin (2009), pp. 39-49.

[13]. Metin Soycan (2014). Improving EGM2008 by GPS and leveling data at local scale, BCG - Boletin de Ciências Geodésicas Sec, Artigos, Curitiba, V.20, No.1, PP. 3-18, on - lineversion, ISSN 1982-2170, http://dx.doi.org/10.1590/S1982-21702014000100001.

[14]. Minoru Tanaka (2000). Periodic Anomalous Vertical Crustall Movement in Tectonic Regions and its Ingluences on Volcanic Activities - Sakarajima Volcano. Rep. Fac. Sci., Kagoshima Univ., No. 33, pp. 89-100.

[15]. Li Jiancheng, Chao Dingbo, Shen Wenbin, Zang Shoujian, Zou Xiancai, Jiang Weiping and Yao Yibin (2011). Progress in Geoid Determination Research Areas in China. In: China National Report on Geodesy (2007-2010) for the XXV General Assembly of IUGG, Melburne, Australia, 27 June - 8 July 2011.

[16]. Pavlis N.K., Factor J.K. and Holmes S.A. (2007). Terrain - related Gravimetric Quantities Computed for the Next EGM. Proceedings of the 1st International Symposium of the International Gravity Field Service (IGFS), Istanbul, pp. 318-323.

[17]. Pavlis Nikolas K, Simon A. Holmes, Steve C. Kenyon, John K. Factor (2008). An Earth gravitational model to degree 2160: EGM2008. EGU General asembly 2008, Vienna, Austria, April 13 - 18, 2008.

[18]. Pavlis N.K., Holmes S.A., Kenyon S.C. and Factor J.K. (2009). The Earth Gravitational Model 2008 (EGM2008). The OSU School of the Earth Sciences, Columbus, $\mathrm{OH}$, February, 12/2009, $51 \mathrm{p}$.

(Xem tiếp trang 25) 